\title{
Stability analysis of $f(R)$-AdS black holes
}

\author{
Taeyoon Moon $*$, Yun Soo Myung 6 t, and Edwin J. Sond \\ ${ }^{a}$ Center for Quantum Space-time, Sogang University, Seoul, 121-742, Korea \\ ${ }^{b}$ Institute of Basic Sciences and School of Computer Aided Science, Inje University \\ Gimhae 621-749, Korea
}

\begin{abstract}
We study the stability of $f(R)$-AdS (Schwarzschild-AdS) black hole obtained from $f(R)$ gravity. In order to resolve the difficulty of solving fourth order linearized equations, we transform $f(R)$ gravity into the scalar-tensor theory by introducing two auxiliary scalars. In this case, the linearized curvature scalar becomes a dynamical scalaron, showing that all linearized equations are second order. Using the positivity of gravitational potentials and $S$-deformed technique allows us to guarantee the stability of $f(R)$-AdS black hole if the scalaron mass squared satisfies the BreitenlohnerFreedman bound. This is confirmed by computing quasinormal frequencies of the scalaron for $f(R)$-AdS black hole.
\end{abstract}

PACS numbers:

Typeset Using $\mathrm{IT}_{\mathrm{E}} \mathrm{X}$

*e-mail address: tymoon@sogang.ac.kr

$\dagger$ e-mail address: ysmyung@inje.ac.kr

$\ddagger$ e-mail address: eddy@sogang.ac.kr 


\section{Introduction}

$f(R)$ gravities [1, 2, 3] have much attention as one of strong candidates for explaining the current accelerating universe [4]. $f(R)$ gravities can be considered as Einstein gravity with an additional scalar. For example, it was shown that the metric- $f(R)$ gravity is equivalent to the $\omega_{\mathrm{BD}}=0$ Brans-Dicke (BD) theory with a certain potential [5]. However, in order that $f(R)$ gravities are acceptable, they obey minimal requirements for theoretical viability [2, 5]. Three important requirements are included: (i) they possess the correct cosmological dynamics, (ii) they are free from instabilities of tachyon and ghost [6, 7, 8], (iii) they attain correct Newtonian and post-Newtonian limits.

The Schwarzschild-de Sitter black hole was first obtained for a positively constant curvature scalar in $f(R)$ gravities [9] and other black hole solution was recently found for a nonconstant curvature scalar in $f(R)$ gravities [10]. A black hole solution was also obtained from $f(R)$ gravities by requiring the negative constant curvature scalar $R=\bar{R}<0$ [11]. For $1+f^{\prime}(\bar{R})>0$, this is similar to the Schwarzschild-AdS (SAdS) black hole obtained from Einstein gravity. In order to obtain the constant curvature black hole from " $f(R)$ gravity coupled to the matter", the trace of its stress-energy tensor $T_{\mu \nu}$ should be zero. Two solutions were found when the Maxwell [11] and Yang-Mills fields [12] are coupled to $f(R)$ gravities.

Gravitational stability of a black hole is a main issue of testing the adequateness of the solution [13, 14. However, the stability analysis seems not directly applicable to $f(R)$ black holes including the Kerr black hole because $f(R)$ gravity contains fourth-order derivative terms in the linearized equations [15, 16]. In this case, the requirement (ii)[they are free from instabilities of tachyon and ghost] would come into play in testing the stability of $f(R)$ black holes [17. One may transform $f(R)$ gravity into the scalar-tensor theory to eliminate fourth-order derivative terms by introducing two scalar fields [18]. Then, the linearized curvature scalar became a scalaron, guaranteeing that all linearized equations are second order. For $f(R)$ black hole, its linearized equations have led to the same equations for the BD theory [19, 20]. Using the stability analysis of black hole in the massive BD theory [20], it was shown that the $f(R)$ black hole is stable against the external perturbations if the scalaron does not have a tachyonic mass in asymptotically flat spacetimes 21.

In this work, we investigate the stability of $f(R)$-AdS (SAdS) black hole. We transform $f(R)$ gravity into the scalar-tensor theory to avoid fourth-order derivative terms by intro- 
ducing two auxiliary scalars. Then, the linearized curvature scalar becomes a scalaron, indicating that all linearized equations are second order. Using the positivity of gravitational potentials and $S$-deformed technique [22] allows us to prove the stability of $f(R)$-AdS black hole if the scalaron mass squared $\left(m_{A}^{2}\right)$ satisfies the Breitenlohner-Freedman (BF) bound $\left(m_{A}^{2} \geq m_{\mathrm{BF}}^{2}=-\frac{9}{4 \ell^{2}}\right)$ [23]. In order to confirm the stability of $f(R)$-AdS black hole, we have to realize that a practical tool for testing stability in all those cases is a numerical investigation of quasinormal spectra $k=k_{R}-i k_{I}$ [13, 24, 25]. We have checked that there is no exponentially growing mode (tachyon instability) in anti de Sitter space if the scalaron mass squared satisfies the Breitenlohner-Freedman bound $\left(m_{A}^{2} \geq m_{\mathrm{BF}}^{2}\right)$. For $m_{A}^{2}<m_{\mathrm{BF}}^{2}$, however, we have found a growing mode, showing the tachyonic instability of $f(R)$-AdS black hole.

\section{Perturbation of $f(R)$-AdS black holes}

Let us first consider $f(R)$ gravity without any matter fields

$$
S_{f}=\frac{1}{2 \kappa^{2}} \int d^{4} x \sqrt{-g} f(R)
$$

with $\kappa^{2}=8 \pi G$. Since all relevant equations derived from (2.1) are given by Ref. [21], we do not reproduce them here for saving the space. The point to remember is that there exist fourth-order linearized equations around the $f(R)$-AdS black holes. In order to avoid it, we introduce two auxiliary fields $\phi$ and $A$ which may allow to rewrite the action (2.1) as [18]

$$
S_{A}=\frac{1}{2 \kappa^{2}} \int d^{4} x \sqrt{-g}[\phi(R-A)+f(A)] .
$$

Varying for the fields $\phi$ and $A$ lead to the following equations:

$$
R=A, \quad \phi=f^{\prime}(A)
$$

where ' denotes differentiation with respect to its argument. Note that imposing (2.3) on the action (2.2) recovers the original action (2.1). On the other hand, the equation of motion for the metric tensor can be obtained by

$$
f^{\prime}(A) R_{\mu \nu}-\frac{f(A)}{2} g_{\mu \nu}+\left(g_{\mu \nu} \nabla^{2}-\nabla_{\mu} \nabla_{\nu}\right) f^{\prime}(A)=0
$$


In deriving the above equation, we used two relations in (2.3). Considering a constant curvature scalar $R=\bar{R}=\bar{A}$ together with $\bar{\phi}=f^{\prime}(\bar{A})=$ const, Eq.(2.4) becomes

$$
f^{\prime}(\bar{A}) \bar{R}_{\mu \nu}-\frac{1}{2} \bar{g}_{\mu \nu} f(\bar{A})=0 .
$$

Taking the trace of (2.5) leads to

$$
\bar{R}=\frac{2 f(\bar{A})}{f^{\prime}(\bar{A})} \equiv 4 \Lambda_{A}
$$

which determines the positive, negative and zero curvature scalar by choosing a specific form of $f(A)$.

At this stage, we wish to point out the difference between $R=A$ in (2.3) and (2.6) . The former is the strong constraint, while the latter is satisfied only in the background of constant curvature scalar. In general, $R$ is determined by the trace equation

$$
R=\frac{-3 \nabla^{2} f^{\prime}(A)+2 f(A)}{f^{\prime}(A)}
$$

Substituting (2.6) into (2.5), one finds the Ricci tensor which determines the maximally symmetric Einstein spaces including Minkowski space

$$
\bar{R}_{\mu \nu}=\frac{1}{2} \frac{f(\bar{A})}{f^{\prime}(\bar{A})} \bar{g}_{\mu \nu}=\Lambda_{A} \bar{g}_{\mu \nu} .
$$

A $f(R)$-AdS black hole solution is given by a spherically symmetric form

$$
d s^{2}=\bar{g}_{\mu \nu} d x^{\mu} d x^{\nu}=-e^{\nu(r)} d t^{2}+e^{-\nu(r)} d r^{2}+r^{2}\left(d \theta^{2}+\sin ^{2} \theta d \varphi^{2}\right)
$$

with

$$
e^{\nu(r)}=1-\frac{2 m}{r}-\frac{\Lambda_{A}}{3} r^{2}
$$

We focus on asymptotically $\mathrm{AdS}_{4}$ spacetime with $\Lambda_{A}=-\frac{3}{\ell^{2}}<0$ and consider the background of

$$
\bar{R}=\bar{A}=2 f(\bar{A}) / f^{\prime}(\bar{A})<0,
$$

with $f(\bar{A})<0$.

Now we introduce the perturbation around the constant curvature black hole to study stability of the $f(R)$-AdS black hole

$$
g_{\mu \nu}=\bar{g}_{\mu \nu}+h_{\mu \nu} .
$$


Hereafter the background quantities are denoted by the "overbar" notation. In this background, we define the perturbations as

$$
\bar{R}+\delta R(h)=\bar{A}+\delta A, \quad f(A)=f(\bar{A})+f^{\prime}(\bar{A}) \delta A, \quad f^{\prime}(A)=f^{\prime}(\bar{A})+f^{\prime \prime}(\bar{A}) \delta A .
$$

The first relation implies the replacement of linearized curvature scalar by the linearized scalaron as

$$
\delta R(h)=R-\bar{R} \rightarrow \delta A=A-\bar{A} .
$$

Also the linearized equation to (2.4) takes the form

$$
\begin{aligned}
\delta R_{\mu \nu}(h) & -\Lambda_{A} h_{\mu \nu}+\bar{g}_{\mu \nu}\left[\frac{f^{\prime \prime}(\bar{A}) f(\bar{A})-f^{\prime 2}(\bar{A})}{2 f^{\prime 2}(\bar{A})}\right] \delta A \\
& +\left[\frac{f^{\prime \prime}(\bar{A})}{f^{\prime}(\bar{A})}\right]\left(\bar{g}_{\mu \nu} \bar{\nabla}^{2}-\bar{\nabla}_{\mu} \bar{\nabla}_{\nu}\right) \delta A=0,
\end{aligned}
$$

where the linearized Ricci tensor $\delta R_{\mu \nu}(h)$ is given by

$$
\delta R_{\mu \nu}(h)=\frac{1}{2}\left(\bar{\nabla}^{\rho} \bar{\nabla}_{\mu} h_{\nu \rho}+\bar{\nabla}^{\rho} \bar{\nabla}_{\nu} h_{\mu \rho}-\bar{\nabla}^{2} h_{\mu \nu}-\bar{\nabla}_{\mu} \bar{\nabla}_{\nu} h\right) .
$$

It is important to note that taking the trace of (2.15) with $\bar{g}^{\mu \nu}$ together with (2.14) leads to the linearized "scalaron" equation as

$$
\left(\bar{\nabla}^{2}-m_{A}^{2}\right) \delta A=0
$$

where the scalaron mass squared $m_{A}^{2}$ is given by

$$
m_{A}^{2}=\frac{f^{\prime 2}(\bar{A})-2 f(\bar{A}) f^{\prime \prime}(\bar{A})}{3 f^{\prime}(\bar{A}) f^{\prime \prime}(\bar{A})}=\frac{f^{\prime}(\bar{A})}{3 f^{\prime \prime}(\bar{A})}-\frac{4}{3} \Lambda_{A},
$$

which was already known as (97) of Ref. 2] in de Sitter spacetimes. Plugging (2.17) into (2.15) and rearranging the terms, we obtain the linearized equation

$$
\delta R_{\mu \nu}(h)-\Lambda_{A} h_{\mu \nu}=\left[\frac{f^{\prime \prime}(\bar{A})}{f^{\prime}(\bar{A})}\right] \bar{\nabla}_{\mu} \bar{\nabla}_{\nu} \delta A+\bar{g}_{\mu \nu}\left[\frac{f^{\prime 2}(\bar{A})+f(\bar{A}) f^{\prime \prime}(\bar{A})}{6 f^{\prime 2}(\bar{A})}\right] \delta A .
$$

Since the mass dimension of the scalaron is two $([\delta A]=2)$, it would be better to write the canonically linearized equations by introducing a dimensionless scalaron $\delta \tilde{A}$ defined by

$$
\delta \tilde{A}=\frac{f^{\prime \prime}(\bar{A})}{f^{\prime}(\bar{A})} \delta A .
$$


Here $\left[f^{\prime}(\bar{A})\right]=0$ and $\left[f^{\prime \prime}(\bar{A})\right]=-2$.

Finally, we arrive at two linearized equations

$$
\begin{aligned}
& \left(\bar{\nabla}^{2}-m_{A}^{2}\right) \delta \tilde{A}=0, \\
& \delta R_{\mu \nu}(h)-\Lambda_{A} h_{\mu \nu}-\bar{\nabla}_{\mu} \bar{\nabla}_{\nu} \delta \tilde{A}-\left[\frac{m_{A}^{2}}{2}+\Lambda_{A}\right] \bar{g}_{\mu \nu} \delta \tilde{A}=0,
\end{aligned}
$$

which should be solved to carry out the stability analysis of $f(R)$-AdS black hole.

\section{Stability analysis of $f(R)$-AdS black hole}

The metric perturbations $h_{\mu \nu}$ are classified depending on the transformation properties under parity, namely odd (axial) and even (polar). Using the Regge-Wheeler [26], and Zerilli gauge [27], one obtains two distinct perturbations : odd and even perturbations. For odd parity, one has with two off-diagonal components $h_{0}$ and $h_{1}$

$$
h_{\mu \nu}^{o}=\left(\begin{array}{cccc}
0 & 0 & 0 & h_{0}(r) \\
0 & 0 & 0 & h_{1}(r) \\
0 & 0 & 0 & 0 \\
h_{0}(r) & h_{1}(r) & 0 & 0
\end{array}\right) e^{-i k t} \sin \theta \frac{d p_{L}}{d \theta}
$$

while for even parity, the metric tensor takes the form with four components $H_{0}, H_{1}, H_{2}$, and $K$ as

$$
h_{\mu \nu}^{e}=\left(\begin{array}{cccc}
H_{0}(r) e^{\nu(r)} & H_{1}(r) & 0 & 0 \\
H_{1}(r) & H_{2}(r) e^{-\nu(r)} & 0 & 0 \\
0 & 0 & r^{2} K(r) & 0 \\
0 & 0 & 0 & r^{2} \sin ^{2} \theta K(r)
\end{array}\right) e^{-i k t} p_{L},
$$

where $p_{L}$ is Legendre polynomial with angular momentum $L$ and $e^{\nu(r)}$ was given by (2.10). In order to achieve the stability of $f(R)$ black hole in asymptotically flat spacetimes, we have used the result for the stability analysis for the massive BD theory [19]. However, the present situation is quite different from $f(R)$ black hole, because we are going to perform the stability analysis of $f(R)$-AdS black hole. 


\subsection{Odd-perturbation}

For odd-parity perturbation, its linearized equation takes a simple form as

$$
\delta R_{\mu \nu}(h)-\Lambda_{A} h_{\mu \nu}=0
$$

Using three equations of $\delta R_{t \varphi}, \delta R_{r \varphi}$, and $\delta R_{\theta \varphi}$ in (3.3) together with (2.16) and (3.1), one finds the following equation for $h_{1}$ :

$$
\frac{d^{2} h_{1}}{d r^{2}}+\left(3 \nu^{\prime}-\frac{2}{r}\right) \frac{d h_{1}}{d r}+\left[\nu^{\prime \prime}+2 \nu^{\prime 2}+k^{2} e^{-2 \nu}-e^{-\nu} \frac{(L-1)(L+2)}{r^{2}}\right] h_{1}=0 .
$$

Introducing the tortoise coordinate $r^{*}=\int e^{-\nu(r)} d r$ which maps $r \in\left[r_{+}, \infty\right]$ into $r^{*} \in$ $[-\infty, 0]$ and a new field $Q$ defined by

$$
Q=e^{\nu} \frac{h_{1}}{r}
$$

(3.4) leads to the Regge-Wheeler equation as

$$
\frac{d^{2} Q}{d r^{* 2}}+\left[k^{2}-V_{R W}\right] Q=0
$$

Here the Regge-Wheeler potential is given by [26, 28]

$$
V_{R W}(r)=\left(1-\frac{2 m}{r}-\frac{\Lambda_{A}}{3} r^{2}\right)\left[\frac{L(L+1)}{r^{2}}-\frac{6 m}{r^{3}}\right] .
$$

It is well known from [28] that the effective potential can be positive definite outside the SAdS black hole when using $S$-deformed technique [22]. This implies that the $f(R)$-AdS black holes are stable against odd-perturbation because (3.6) is equivalent to that of the SAdS black hole in Einstein gravity.

\subsection{Even-perturbation}

We consider the linearized equation (2.22) fully to perform even-parity perturbation. Considering the metric perturbation (3.2) and the scalar perturbation given by

$$
\delta \tilde{A} \propto \Sigma \frac{\psi(r)}{r} Y_{L}^{M}(\theta, \varphi) e^{-i k t}
$$

with $Y_{L}^{M}(\theta, \varphi)$ spherical harmonics, the equation of either $(\theta \theta)$ or $(\varphi \varphi)$ component yields

$$
H_{2}=H_{0}-2 \frac{\psi}{r} .
$$


From five remaining equations of $(t r),(r r),(t t),(t \theta)$, and $(r \theta)$ components, one obtains the constraint equation

$$
\begin{aligned}
& \left\{\tilde{L}^{2}-2 e^{\nu}+r e^{\nu} \nu^{\prime}\right\} H_{0}+\left\{2 k^{2} r^{2} e^{-\nu}+2 e^{\nu}+r e^{\nu} \nu^{\prime}+\frac{1}{2} r^{2} e^{\nu} \nu^{\prime 2}-\tilde{L}^{2}+2 \Lambda_{A} r^{2}\right\} K \\
& -\left\{2 i k r+\frac{\tilde{L}^{2}}{2 i k}\right\} H_{1}+\left\{2 \tilde{L}^{2}-4 e^{\nu}-2 k^{2} r^{2} e^{-\nu}+\frac{1}{2} r^{2} e^{\nu} \nu^{\prime 2}-2 \Lambda_{A} r^{2}\right\} \frac{\psi}{r}=0
\end{aligned}
$$

with $\tilde{L}^{2}=L(L+1)$. In deriving this expression, we have used $\nu^{\prime \prime}+\left(\nu^{\prime}\right)^{2}+2 \nu^{\prime} / r=-2 \Lambda_{A} e^{-\nu}$. At this stage, we introduce the tortoise coordinate $\left(r^{*}\right)$ and a new field defined by

$$
\hat{M}=\frac{1}{p q-h}\left\{p\left(K+\frac{\psi}{r}\right)-\frac{H_{1}}{k}\right\}
$$

where

$$
\begin{aligned}
& q(r)=\frac{\lambda(\lambda+1) r^{2}+3 \lambda m r+6 m^{2}}{r^{2}(\lambda r+3 m)}, \quad h(r)=\frac{i\left(-\lambda r^{2}+3 \lambda m r+3 m^{2}\right)}{\left(r-2 m-\frac{\Lambda_{A}}{3} r^{3}\right)(\lambda r+3 m)}, \\
& p(r)=-\frac{i r^{2}}{r-2 m-\frac{\Lambda_{A}}{3} r^{3}}, \quad \lambda=\frac{1}{2}(L-1)(L+2) .
\end{aligned}
$$

Manipulating $(t r)$ and $(t \theta)$ component equations in (2.22), we arrive at the Zerilli's equation 1

$$
\frac{d^{2} \hat{M}}{d r^{* 2}}+\left[k^{2}-V_{Z}\right] \hat{M}=0,
$$

where the Zerilli potential is given by [27, 28]

$$
V_{Z}(r)=\left(1-\frac{2 m}{r}-\frac{\Lambda_{A}}{3} r^{2}\right)\left[\frac{2 \lambda^{2}(\lambda+1) r^{3}+6 \lambda^{2} m r^{2}+18 \lambda m^{2} r+18 m^{3}-6 \Lambda_{A} m^{2} r^{3}}{r^{3}(\lambda r+3 m)^{2}}\right] .
$$

The Zerilli potential $V_{Z}$ is always positive for whole range of $-\infty \leq r^{*} \leq 0$ (see Fig. 9 of [22]), which implies that the even-perturbation is stable, even though the scalaron $\psi(\delta \tilde{A})$ is coupled to making the even-perturbation.

\footnotetext{
${ }^{1}$ At this stage, one may ask the question of "can one find the single metric components (e.g. $H_{1}$ or $\psi$ ) from the master variable $\hat{M}$ ?". The answer is obviously "No". Actually, one has $h_{\mu \nu}$ with 10 components in Einstein gravity. A massless graviton has 2 degrees of freedom (DOF). Upon the Regge-Wheeler gaugefixing, one has 6 . One DOF is given by the odd-perturbation combined by $2\left(h_{1}\right.$ and $\left.h_{2}\right)$ and the other is the even-perturbation combined by $4\left(H_{0}, H_{1}, H_{2}, K\right)$. In $f(R)$ gravities, one starts with 11 (10 from $h_{\mu \nu}$ and 1 from $\psi$ ), indicating 3 DOF (massless graviton and scalaron) totally. Hence, the scalaron should be coupled to giving the master variable $\hat{M}$ in the even-perturbation.
} 


\subsection{Scalar-perturbation}

The main difference between Einstein and $f(R)$ gravities is the appearance of scalaron. Hence, the stability analysis of scalaron equation will be considered to be the main part of the present work. In order to obtain the second-order differential equation for the scalaron, we first recall that the scalaron perturbation is given by (3.8). In this case, Eq.(2.21) becomes

$$
\frac{e^{\nu}}{r} \psi^{\prime \prime}+\frac{e^{\nu} \nu^{\prime}}{r} \psi^{\prime}-\left[\frac{m_{A}^{2}-e^{-\nu} k^{2}}{r}+\frac{e^{\nu} \nu^{\prime}}{r^{2}}+\frac{L(L+1)}{r^{3}}\right] \psi=0
$$

Using the tortoise coordinate $\left(r^{*}\right)$, the above equation reduces to the Schrödinger-type equation

$$
\frac{d^{2} \psi}{d r^{* 2}}+\left[k^{2}-V_{A}\right] \psi=0,
$$

where the scalaron potential $V_{A}$ is given by

$$
V_{A}(r)=\left(1-\frac{2 m}{r}-\frac{\Lambda_{A}}{3} r^{2}\right)\left[\frac{L(L+1)}{r^{2}}+\frac{2 m}{r^{3}}-\frac{2}{3} \Lambda_{A}+m_{A}^{2}\right] .
$$

Here, the second term in square bracket is the usual scalar term with spin zero [in general, $-2 m\left(s^{2}-1\right)$ for $s$ spin-weight of a perturbing field], and the third arises from $\mathrm{AdS}_{4}$ asymptote. Importantly, the last term in bracket shows the feature of a massive scalaron arisen from $f(R)$ gravity. This term could not be eliminated using the $S$-deformed technique because it is not an element of the metric function $e^{\nu(r)}$. Furthermore, it is well known that there exists the BF bound [23] for the Klein-Gordon type equation (2.17)

$$
m_{A}^{2} \geq m_{\mathrm{BF}}^{2}=-\frac{9}{4 \ell^{2}}
$$

which implies an inequality

$$
\frac{f^{\prime}(\bar{A})}{3 f^{\prime \prime}(\bar{A})} \geq-\frac{25}{4 \ell^{2}}
$$

On the other hand, we rewrite the scalaron potential (3.17)

$$
V_{A}(r)=\left(1-\frac{2 m}{r}-\frac{\Lambda_{A}}{3} r^{2}\right)\left[\frac{L(L+1)}{r^{2}}+\frac{2 m}{r^{3}}+\frac{6}{\ell^{2}}+\frac{f^{\prime}(\bar{A})}{3 f^{\prime \prime}(\bar{A})}\right],
$$

which shows the asymptotic positivity $\left(V_{A}(r \rightarrow \infty) \rightarrow 0\right)$ when the following condition is achieved

$$
\frac{f^{\prime}(\bar{A})}{3 f^{\prime \prime}(\bar{A})} \geq-\frac{6}{\ell^{2}}
$$


Hence, we allow the negative mass squared in $\mathrm{AdS}_{4}$ spacetimes which belongs to the region

$$
-\frac{25}{4 \ell^{2}} \leq \frac{f^{\prime}(\bar{A})}{3 f^{\prime \prime}(\bar{A})} \leq-\frac{6}{\ell^{2}} \rightarrow-\frac{9}{4 \ell^{2}} \leq m_{A}^{2} \leq-\frac{2}{\ell^{2}}
$$

We note that the left bound comes from the BF bound, while the right bound arises from the asymptotic positivity condition of $V_{A}(r \rightarrow \infty) \rightarrow 0$. Accordingly, we divide $m_{A}^{2}$ into four region:2: $m_{A}^{2} \ell^{2}<m_{\mathrm{BF}}^{2} \ell^{2}=-9 / 4, \quad-9 / 4 \leq m_{A}^{2} \ell^{2} \leq-2, \quad-2<m_{A}^{2} \ell^{2}<0$, and $m_{A}^{2} \ell \geq 0$. The boundaries are $m_{A}^{2} \ell^{2}=0,-2,-9 / 4$. As is shown in Fig. 1 , there are four types of graphs including three boundaries, depending on the mass squared $m_{A}^{2}$. This may imply that there is no ghost instability even for the negative mass squared is allowed, if $m_{A}^{2}$ satisfies the bound (3.22). This is surely a new feature of stability condition for $f(R)$-AdS black holes. On the other hand, we expect that for $m_{A}^{2}<m_{\mathrm{BF}}^{2}$, there will be the tachyonic instability.

\section{Quasinormal modes}

It is well known that a practical tool for testing stability of all kinds of black holes is a numerical investigation of the quasinormal spectra [13]. Hence, we wish to confirm the stability of $f(R)$-AdS black holes by computing quasinormal modes. Considering $\Psi\left(t, r_{*}\right)=$ $e^{-i k t} \Psi\left(r_{*}\right)$, the boundary conditions for quasinormal modes propagating in the $f(R)$-AdS black hole background are given by

$$
\Psi\left(r_{*}\right) \sim \begin{cases}e^{-i k r_{*}}, & \text { for } r_{*} \rightarrow-\infty, \\ 0, & \text { for } r_{*} \rightarrow 0,\end{cases}
$$

where $\Psi\left(r_{*}\right)$ represents $Q$ (odd), $\hat{M}$ (even), and $\psi$ (scalaron) in (3.6), (3.13), and (3.16), respectively. The frequencies of the quasinormal modes are given by complex numbers,

\footnotetext{
${ }^{2}$ The fourth region $m_{A}^{2} \ell^{2} \geq 0$ corresponds to "tachyon-free condition" required by the stability of de Sitter space of $\left(f^{\prime 2}-2 f f^{\prime \prime}\right) / 3 f^{\prime} f^{\prime \prime} \geq 0$ [7, 8, 29, 30] and Minkowski space of $f^{\prime} / 3 f^{\prime \prime} \geq 0$ [18. This implies that $f^{\prime}>0$ and $f^{\prime \prime}>0$ for stability. However, the stable region (tachyon-free case) could be extended to negative mass squared in the AdS space: $m_{A}^{2} \ell^{2} \geq-9 / 4[(\underline{3.19})]$, which implies that $f^{\prime \prime}(\bar{A})<0$, for $f^{\prime}(\bar{A})>0$ in AdS space. This is in contrast with other perturbation analysis: the Dolgov-Kawasaki instability with $f^{\prime \prime}<0$ in cosmological perturbations [31, graviton and scalar propagations in the Minkowski [18, 32].
} 


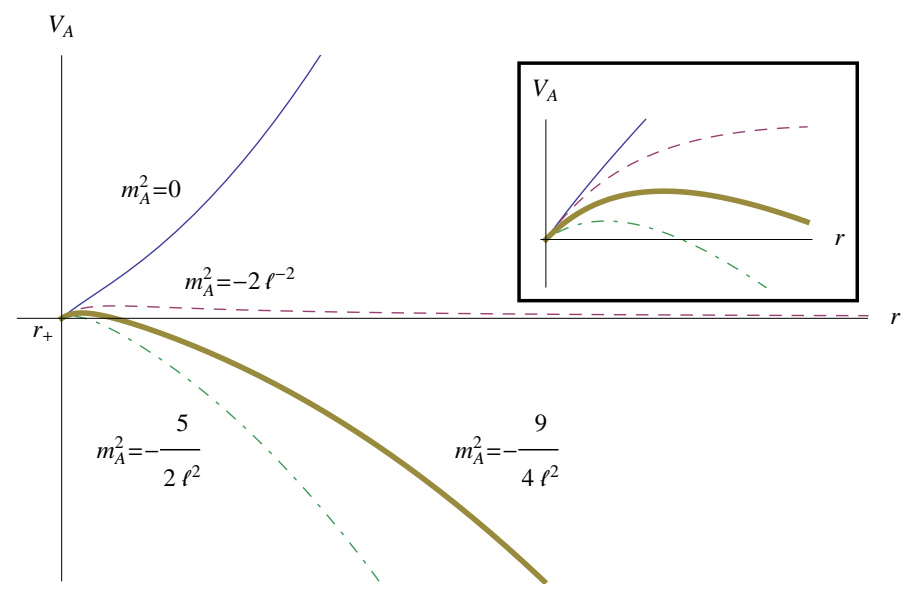

Figure 1: Scalaron potential $V_{A}$ graphs as function of $r$ with fixed $\ell^{2}=1$ : for $m_{A}^{2} \ell^{2}=0$ case, it shows the massless scalaron in AdS spacetimes, while for $m_{A}^{2} \ell^{2}=-2$ case, it approaches zero as $r \rightarrow \infty$ (asymptotic positivity). For two cases of $m_{A}^{2} \ell^{2}=-9 / 4$ and $-5 / 2$, their potentials approach negative infinity as $r \rightarrow \infty$.

$k=k_{R}-i k_{I}$, and the modes with $k_{I}>0\left(k_{I}<0\right)$ corresponding to decaying (growing) ones, since the time dependence is given by $e^{-i k t}=e^{-k_{I} t} e^{-i k_{R} t}$. We note that if there is at least one growing mode, the black hole is unstable.

Now, (3.6), (3.13), and (3.16) could be written as

$$
\frac{d^{2} \Psi\left(r_{*}\right)}{d r_{*}^{2}}+\left(k^{2}-V\right) \Psi\left(r_{*}\right)=0
$$

with $V$ denoting $V_{R W}, V_{Z}$, and $V_{A}$. Multiplying this equation by a complex conjugated function $\bar{\Psi}\left(r_{*}\right)$ and integrating it, we have

$$
\begin{aligned}
I & =\int_{-\infty}^{0} d r_{*}\left[\bar{\Psi}\left(r_{*}\right) \frac{d^{2} \Psi\left(r_{*}\right)}{d r_{*}^{2}}+\left(k^{2}-V\right)\left|\Psi\left(r_{*}\right)\right|^{2}\right] \\
& =i k|\Psi(-\infty)|^{2}+\int_{-\infty}^{0} d r_{*}\left[-\left|\frac{d \Psi\left(r_{*}\right)}{d r_{*}}\right|^{2}+\left(k^{2}-V\right)\left|\Psi\left(r_{*}\right)\right|^{2}\right]=0,
\end{aligned}
$$

where we used the boundary condition (4.1). The imaginary part of (4.3) takes the form

$$
\operatorname{Im}(I)=k_{R}\left[|\Psi(-\infty)|^{2}-2 k_{I} \int_{-\infty}^{0} d r_{*}\left|\Psi\left(r_{*}\right)\right|^{2}\right]=0,
$$

which implies that either $k_{R}=0$ or $k_{I}>0$, so that the growing modes cannot oscillate as was pointed out in [25, 13. That is, the unstable mode is defined by the condition of

$$
\text { unstable mode } \rightarrow k_{R}=0, \quad k_{I}<0 .
$$


in the quasinormal-mode approach. Next, the real part of (4.3) is given by

$$
\begin{aligned}
\operatorname{Re}(I)= & k_{I}|\Psi(-\infty)|^{2}+\left(k_{R}^{2}-k_{I}^{2}\right) \int_{-\infty}^{0} d r_{*}\left|\Psi\left(r_{*}\right)\right|^{2} \\
& -\int_{-\infty}^{0} d r_{*}\left[\left|\frac{d \Psi\left(r_{*}\right)}{d r_{*}}\right|^{2}+V\left|\Psi\left(r_{*}\right)\right|^{2}\right]=0
\end{aligned}
$$

which means that the unstable mode does not exist if the potential $V$ is positive definite. We confirm that there is no unstable modes for the even-perturbation since $V_{Z}$ is always positive. For the odd-perturbation, $V_{R W}$ becomes negative near the horizon, but the stability may be achieved by the $S$-deformation technique. On the other hand, it turned out that there is no growing modes in odd- and even-quasinormal mode computations [28, 33]. Hence, we do not need to compute quasinormal frequencies for the metric perturbations again.

However, we remind the reader the instability of the scalar perturbation depends on the scalaron mass squared, $m_{A}^{2}$. Requiring $V_{A}$ to be positive corresponds to the bound of $m_{A}^{2} \geq-2 \ell^{-2}$. Thus, one expects that there is no exponentially growing mode for this case. Also, if $m_{A}^{2}$ satisfies the BF bound $\left(m_{\mathrm{BF}}^{2} \leq m_{A}^{2} \leq-2 \ell^{-2}\right)$, there is no tachyon instability. Finally, if $m_{A}^{2}<m_{\mathrm{BF}}^{2}$, there will be tachyonic instability. In order to show it explicitly, we calculate quasinormal frequencies of the scalaron numerically.

To this end, Eq. (4.2) should be rewritten in the ingoing Eddington coordinates with $v=t+r_{*}$, following the original work of Ref. 34] for large Schwarzschild-AdS black holes:

$$
e^{\nu} \frac{d^{2} \tilde{\Psi}}{d r^{2}}+\left(\nu^{\prime} e^{\nu}-2 i k\right) \frac{d \tilde{\Psi}}{d r}-\tilde{V} \tilde{\Psi}=0
$$

where $\Psi\left(t, r_{*}\right)=e^{-i k v} \tilde{\Psi}$ and $V=e^{\nu} \tilde{V}$. Then, changing coordinate variable $r$ into $x=\ell / r$, the equation (4.7) becomes

$$
s(x) \frac{d^{2} \tilde{\Psi}}{d x^{2}}+\frac{t(x)}{x-x_{+}} \frac{d \tilde{\Psi}}{d x}+\frac{u(x)}{\left(x-x_{+}\right)^{2}} \tilde{\Psi}=0,
$$

where

$$
\begin{aligned}
& s(x)=\frac{x^{4} e^{\nu}}{x_{+}-x}=\frac{x^{2}}{x_{+}^{3}}\left[x_{+}^{2}+x_{+} x+\left(1+x_{+}^{2}\right) x^{2}\right]=\sum_{n=0}^{4} s_{n}\left(x-x_{+}\right)^{n}, \\
& t(x)=-2 i \tilde{k} x^{2}-2 x^{3}+\frac{3}{x_{+}^{3}}\left(1+x_{+}^{2}\right) x^{4}=\sum_{n=0}^{4} t_{n}\left(x-x_{+}\right)^{n}, \\
& u(x)=\left(x-x_{+}\right) \tilde{V}=\sum_{n=0}^{4} u_{n}\left(x-x_{+}\right)^{n} .
\end{aligned}
$$


Table 1: Frequencies of quasinormal modes of large $f(R)$-AdS black holes for the scalaron

\begin{tabular}{|c|c|c|c|c|c|c|}
\hline \multirow{2}{*}{$m_{A}^{2} \ell_{+} / \ell$} & \multicolumn{2}{|c|}{10} & \multicolumn{2}{|c|}{50} & \multicolumn{2}{c|}{100} \\
\cline { 2 - 7 } & $\tilde{k}_{R}$ & $\tilde{k}_{I}$ & $\tilde{k}_{R}$ & $\tilde{k}_{I}$ & $\tilde{k}_{R}$ & $\tilde{k}_{I}$ \\
\hline 0 & 18.6070 & 26.6418 & 92.4937 & 133.193 & 184.953 & 266.386 \\
$-1 / 4$ & 18.0708 & 25.6542 & 89.8263 & 128.258 & 179.619 & 256.515 \\
$-1 / 2$ & 17.500 & 24.6056 & 86.988 & 123.018 & 173.945 & 246.036 \\
$-3 / 4$ & 16.888 & 23.4827 & 83.941 & 117.407 & 167.85 & 234.813 \\
-1 & 16.222 & 22.2661 & 80.629 & 111.327 & 161.23 & 222.653 \\
$-5 / 4$ & 15.486 & 20.9261 & 76.968 & 104.630 & 153.91 & 209.260 \\
$-3 / 2$ & 14.64 & 19.42 & 72.81 & 97.067 & 145.6 & 194.13 \\
$-7 / 4$ & 13.66 & 17.63 & 67.88 & 88.15 & 135.7 & 176.3 \\
-2 & 12.3592 & 15.3307 & 61.4106 & 76.6646 & 122.797 & 153.330 \\
$-9 / 4$ & 9.9 & 12 & 49 & 58 & 98 & 116 \\
$-5 / 2$ & 0 & -8.64855 & 0 & -32.5878 & 0 & -59.5519 \\
$-11 / 4$ & 0 & -52.5789 & 0 & -225.272 & 0 & -467.935 \\
-3 & 0 & -126.996 & 0 & -536.309 & 0 & -1035.61 \\
\hline
\end{tabular}

Here $x_{+}=\ell / r_{+}$and $\tilde{k}=k \ell$, and the boundary condition (4.1) takes the form of $\tilde{\Psi}(x \rightarrow$ $0) \rightarrow 0$. Considering a series solution

$$
\tilde{\Psi}=\sum_{n=0}^{\infty} a_{n}\left(x-x_{+}\right)^{n},
$$

the boundary condition at infinity yields an algebraic equation

$$
\sum_{n=0}^{\infty} a_{n}\left(-x_{+}\right)^{n}=0 .
$$

Solving this, one could find (discrete) quasinormal modes. In Table 1, we list quasinormal modes for the scalaron $(L=0)$ obtained by truncating the series after 140 terms. The first row of $m_{A}^{2} \ell^{2}=0$ indicates the quasinormal mode for the massless scalar, which is consistent with Ref. [34]. The last three rows in Table 1 shows clearly that there exist unstable modes of $k_{I}<0$ with $k_{R}=0$ for the scalaron mass $\left(m_{A}^{2} \ell^{2}=-5 / 2<-9 / 4\right)$ below the BF bound. This corresponds to the tachyonic instability. For $-9 / 4 \leq m_{A}^{2} \ell^{2} \leq-2$, there is no unstable 
mode, showing that there is no tachyon instability. This confirms the presence of the BF bound even for $f(R)$-AdS black holes.

\section{Discussions}

We have investigated the stability of $f(R)$-AdS (Schwarzschild-AdS) black hole obtained from the $f(R)$ gravity. Even though the Schwarzschild-AdS black hole is known to be stable in Einstein gravity, checking the stability of $f(R)$-AdS black hole is a nontrivial task because the linearized Einstein equation is fourth order in $f(R)$ gravity. In order to resolve this difficulty, we have translated the fourth-order equation into the second-order equation by introducing auxiliary scalar fields $A$ and $\phi$. In this case, the linearized curvature scalar $\delta R(h)$ becomes a massive scalaron $\delta A$, showing that all linearized equations are second order.

The stability on the metric perturbations remains unchanged, confirming that the odd (even) perturbations lead to the Schrödinger-type equation with the Regge-Wheeler (Zerilli) potentials in asymptotically $\mathrm{AdS}_{4}$ spacetimes. Actually, this corresponds to the case in Einstein gravity, even though the even-perturbation contains the scalaron in addition to $H_{0}, H_{1}, H_{2}$, and $K$. For the odd-perturbation, we have needed the $S$-deformation method to prove the stability of $f(R)$-AdS black hole because the corresponding potential becomes negative near the event horizon.

The main difference comes from the linearized scalaron equation because the scalaron plays the role of a massive scalar, which is physically propagating on the $f(R)$-AdS black hole background. We have shown that $f(R)$-AdS black hole is stable against the scalaronperturbation if the scalaron mass squared $m_{A}^{2}$ satisfies the BF bound. Especially for $-9 / 4 \leq$ $m_{A}^{2} \leq-2$, there is no unstable mode (exponentially growing mode), showing that there is no tachyon instability. This confirms the presence of the BF bound even for $f(R)$-AdS black holes. This is consistent with graviton and scalar propagations AdS [35] spacetimes, but contrasts to other perturbation analysis: the Dolgov-Kawasaki instability with $f^{\prime \prime}(R)<0$ in cosmological perturbations [31, graviton and scalar propagations in the Minkowski [18, 32, and de Sitter spacetimes [29, 30].

Finally, we have found the tachyonic instability for $m_{A}^{2}<m_{\mathrm{BF}}^{2}$. This is confirmed by computing quasinormal frequencies of the scalaron for large $f(R)$-AdS black hole. The last 
three rows in Table 1 shows clearly that there exist unstable modes of $k_{I}<0$ with $k_{R}=0$ for the scalaron mass $\left(m_{A}^{2} \ell^{2}=-5 / 2<-9 / 4\right)$ below the BF bound. This indicates the tachyonic instability.

\section{Acknowledgments}

T. Moon and E. Son were supported by the National Research Foundation of Korea (NRF) grant funded by the Korea government (MEST) through the Center for Quantum Spacetime (CQUeST) of Sogang University with grant number 2005-0049409. Y. Myung was supported by the National Research Foundation of Korea (NRF) grant funded by the Korea government (MEST) (No.2011-0027293).

\section{References}

[1] S. Nojiri and S. D. Odintsov, eConf C0602061 (2006) 06 [Int. J. Geom. Meth. Mod. Phys. 4, 115 (2007)] arXiv:hep-th/0601213.

[2] T. P. Sotiriou and V. Faraoni, Rev. Mod. Phys. 82, 451 (2010) arXiv:0805.1726 [gr$\mathrm{qc}]]$.

[3] S. Nojiri and S. D. Odintsov, Phys. Rept. 505, 59 (2011) arXiv:1011.0544 [gr-qc]].

[4] S. Perlmutter et al. [Supernova Cosmology Project Collaboration], Astrophys. J. 517 (1999) 565 arXiv:astro-ph/9812133;

A. G. Riess et al. [Supernova Search Team Collaboration], Astron. J. 116 (1998) 1009 arXiv:astro-ph/9805201].

[5] A. De Felice and S. Tsujikawa, Living Rev. Rel. 13, 3 (2010) arXiv:1002.4928 [gr-qc]].

[6] L. Pogosian and A. Silvestri, Phys. Rev. D 77 (2008) 023503 [Erratum-ibid. D 81, 049901 (2010)] [arXiv:0709.0296 [astro-ph]].

[7] G. Cognola and S. Zerbini, arXiv:0811.2714 [gr-qc].

[8] S. Capozziello, M. De Laurentis and V. Faraoni, arXiv:0909.4672 [gr-qc]. 
[9] G. Cognola, E. Elizalde, S. Nojiri, S. D. Odintsov and S. Zerbini, JCAP 0502, 010 (2005) arXiv:hep-th/0501096.

[10] L. Sebastiani and S. Zerbini, Eur. Phys. J. C 71, 1591 (2011) [arXiv:1012.5230 [gr-qc]].

[11] A. de la Cruz-Dombriz, A. Dobado and A. L. Maroto, Phys. Rev. D 80, 124011 (2009) arXiv:0907.3872 [gr-qc]]; Phys. Rev. D 83, 029903(E) (2011).

[12] T. Moon, Y. S. Myung and E. J. Son, arXiv:1101.1153 [gr-qc].

[13] R. A. Konoplya and A. Zhidenko, arXiv:1102.4014 [gr-qc].

[14] C. V. Vishveshwara, Phys. Rev. D 1, 2870 (1970).

[15] D. Psaltis, D. Perrodin, K. R. Dienes, and I. Mocioiu, Phys. Rev. Lett. 100, 091101 (2008) arXiv:0710.4564 [astro-ph]].

[16] E. Barausse and T. P. Sotiriou, Phys. Rev. Lett. 101, 099001 (2008) arXiv:0803.3433 [gr-qc]].

[17] A. De Felice, T. Suyama and T. Tanaka, Phys. Rev. D 83, 104035 (2011) arXiv:1102.1521 [gr-qc]].

[18] G. J. Olmo, Phys. Rev. Lett. 95, 261102 (2005) arXiv:gr-qc/0505101.

[19] O. J. Kwon, Y. D. Kim, Y. S. Myung, B. H. Cho and Y. J. Park, Int. J. Mod. Phys. A 1, 709 (1986).

[20] O. J. Kwon, Y. D. Kim, Y. S. Myung, B. H. Cho and Y. J. Park, Phys. Rev. D 34, 333 (1986).

[21] Y. S. Myung, T. Moon and E. J. Son, Phys. Rev. D 83, 124009 (2011) arXiv:1103.0343 $[\mathrm{gr}-\mathrm{qc}]]$.

[22] A. Ishibashi and H. Kodama, Prog. Theor. Phys. 110, 901 (2003) arXiv:hep-th/0305185.

[23] P. Breitenlohner and D. Z. Freedman, Annals Phys. 144, 249 (1982). 
[24] E. Berti, V. Cardoso and A. O. Starinets, Class. Quant. Grav. 26, 163001 (2009) arXiv:0905.2975 [gr-qc]].

[25] R. A. Konoplya, K. Murata, J. Soda and A. Zhidenko, Phys. Rev. D 78, 084012 (2008) arXiv:0807.1897 [hep-th]].

[26] T. Regge and J. A. Wheeler, Phys. Rev. 108, 1063 (1957).

[27] F. J. Zerilli, Phys. Rev. Lett. 24, 737 (1970).

[28] V. Cardoso and J. P. S. Lemos, Phys. Rev. D 64, 084017 (2001) arXiv:gr-qc/0105103.

[29] V. Faraoni and S. Nadeau Phys. Rev. D 72, 124005 (2005) arXiv:gr-qc/0511094.

[30] G. J. Olmo, Phys. Rev. D 75, 023511 (2007) arXiv:gr-qc/0612047.

[31] A. D. Dolgov and M. Kawasaki, Phys. Lett. B 573, 1 (2003) arXiv:astro-ph/0307285.

[32] G. J. Olmo, Phys. Rev. D 72, 083505 (2005).

[33] V. Cardoso, R. Konoplya and J. P. S. Lemos, Phys. Rev. D 68, 044024 (2003) arXiv:gr-qc/0305037].

[34] G. T. Horowitz and V. E. Hubeny, Phys. Rev. D 62, 024027 (2000) arXiv:hep-th/9909056].

[35] Y. S. Myung, Eur. Phys. J. C 71, 1550 (2011) arXiv:1012.2153 [gr-qc]]. 